\title{
Direct Measurement of Hydrodynamic Cross Correlations between Two Particles in an External Potential
}

\author{
Jens-Christian Meiners and Stephen R. Quake \\ Department of Applied Physics, California Institute of Technology, Pasadena, California 91125
}

(Received 22 October 1998)

\begin{abstract}
We report a direct measurement of the hydrodynamic interaction between two colloidal particles. Two micron-sized latex beads were held at varying distances in optical tweezers while their Brownian displacements were measured. In spite of the fact that fluid systems at low Reynolds number are generally considered to have no "memory," the cross-correlation function of the bead positions shows a pronounced, time-delayed anticorrelation. We show that the anticorrelations can be understood in terms of the standard Oseen tensor hydrodynamic coupling. [S0031-9007(99)08607-X]
\end{abstract}

PACS numbers: 82.70.Dd, 83.10.Pp, 87.80.Cc

Hydrodynamic interactions play a crucial role in many physically interesting systems, including colloidal suspensions, polymers in solution, and the microscopic dynamics of proteins. Colloids have a collective diffusion constant that is affected by the distribution of neighboring particles [1], while hydrodynamics interactions are a crucial ingredient in the theory of polymer dynamics [2]. Solvent hydrodynamic effects also have a strong influence on the microscopic dynamics and collective excitations of protein molecules $[3,4]$. It has been shown that secondary structural elements of a protein can move as collective groups [5]. Thus protein molecules have been treated as deformable Brownian particles, which are subject to friction and random forces from the surrounding solvent. Using such a model, Kitao et al. [6] found that, in particular, the dynamics of the low-frequency eigenmodes depend crucially on hydrodynamic effects. Furthermore, such hydrodynamic interactions are thought to play a key role in "steering" ligand-protein binding [7]. Experimentally, it can often be difficult to isolate the effects due to hydrodynamics since measurements are made on bulk systems with indirect methods.

Here we describe an experiment in which we directly studied hydrodynamic interactions between individual colloidal particles. Two microscopic latex beads were held a fixed distance apart in separate optical tweezers. The position fluctuations of the beads were measured, from which we calculated correlation and crosscorrelation functions. Previous studies have used similar arrangements to study electrostatic forces between particles $[8,9]$ and to measure the mutual diffusion constants of two particles [10]. We use it as a simple model system with which to study in detail the effects of hydrodynamic interactions between two particles. The tweezers function as harmonic potential wells and can thus approximate a variety of possible local forces. For example, one can imagine this system idealizing the dynamic motion of two subunits on a large protein complex.

The most striking feature of the experimental data is the presence of a pronounced time-delayed dip in the cross correlations. While it is counterintuitive that the motion of the spheres is anticorrelated, the time delay is also surprising in light of the fact that in fluid systems at low Reynolds number, dynamics are determined only by the instantaneous forces; there is no "memory" [11]. Furthermore, the hydrodynamic interaction does not introduce a propagation delay; it is represented by the Oseen tensor, which is derived directly from the Navier-Stokes equation and assumes instantaneous propagation of forces through the fluid. However, we show here that a stationary, timeindependent external potential can impose time-delayed correlations between particles in solution and that one particle does "remember" where the other one was a short time before. The time delay is determined by the natural relaxation time of the harmonic well.

The notion of memory in these systems can be made precise in the formal context of control theory and linear systems. The concept of "observability" is a mathematical measure of whether or not a system has memory, i.e., whether its complete internal state at some point in the past can be determined from a measurement of its input and output variables [12]. Consider the case of two independent particles in potential wells in which one can measure the position of only one particle. Even if one knows the Brownian forces, the position of the second particle can never be calculated and the system is unobservable. However, introducing hydrodynamic coupling renders the system formally observable, and it is possible to calculate the position of the second particle solely from measurements of the first particle and knowledge of the Brownian forces. The past history of the second particle is encoded in the position of the first.

Experimentally, we studied an aqueous solution of fluorescent carboxyl-modified polystyrene latex spheres with a diameter of $1.0 \pm 0.025 \mu \mathrm{m}$ at a volume fraction of $\phi=10^{-7}$. At such a low concentration additional spheres are typically several hundred $\mu \mathrm{m}$ away from the trapped beads and thus do not interfere with the measurement either by hydrodynamically coupling to the beads or by diffusing into the traps. For some experimental 
runs, $1 \mathrm{M}$ of $\mathrm{NaCl}$ was added to the solution to discriminate between hydrodynamic interactions and possible effects of surface charges. However, no difference in the data obtained from either solution was discernible. This is consistent with the fact that although van der Waals and Coulombic forces are significant at this experiment's force sensitivity, they do not vary appreciably over the distance the beads move in their traps and thus do not contribute to the cross correlations. The solutions were hermetically sealed in a sample cell with a depth of approximately $100 \mu \mathrm{m}$ and a width of $18 \mathrm{~mm}$. The optical potential was applied by means of a dual-beam optical tweezers apparatus. Two orthogonally polarized beams from an Nd:YAG laser at $\lambda=1064 \mathrm{~nm}$ with an intensity of $80 \mathrm{~mW}$ each were focused with an immersion-oil microscope objective (Olympus PlanApo $60 \times 1.4)$ into the sample, with the focal plane lying approximately at a depth of $20 \mu \mathrm{m}$ inside the sample cell. Each of the laser beams holds one of the microspheres in its focus, providing the harmonic potential wells for our experiment. The lateral separation between focal spots and thus the mean separation $E$ between the particles along the $x$ axis was varied between 2 and $15 \mu \mathrm{m}$. The position of the beads was measured by imaging the light scattered from the spheres onto quadrant photodiodes. For this purpose, a microscope objective $(20 \times 0.4)$ is placed on the other side of the sample cell. A polarizing beam splitter separates the light from the two traps before it is focused onto the quadrant photodiodes. A sketch of the apparatus is shown in Fig. 1. To reduce the polarization cross talk and interference phenomena between the two traps, the two trapping beams are chopped alternately at a frequency of $100 \mathrm{kHz}$. Synchronous data acquisition yields positional data for each of the particles that were contaminated by less than a few parts per thousand from cross talk between the traps. Typically, $10^{7}$ data points representing the position of the particles in their traps were acquired at a rate of $50 \mathrm{kHz}$ for each measurement, allowing us to measure forces as low as $10 \mathrm{f} \mathrm{N}$. Subsequent data processing consisted of subtraction of a base line stemming from the dark current of the photodiode and normalization by the photodiode sum intensity to account for laser power fluctuations. Eventually, the autocorrelation functions for each of the particles as well as their cross correlation was calculated. From the latter, an offset resulting from longterm drifts of the experimental apparatus was subtracted. Representative correlation functions are shown in Fig. 2.

The optical traps were calibrated by measuring the autocorrelation function of a bead in one trap with the other trap empty. One expects to find an exponential relaxation whose time constant $\tau_{x}$ is the friction coefficient of the bead divided by the lateral spring constant $k$ of the trap. The friction coefficient is known to within a few percent, and thus the trap strength can be determined. The spring constants of the traps were balanced to within a few percent. In the experimen-

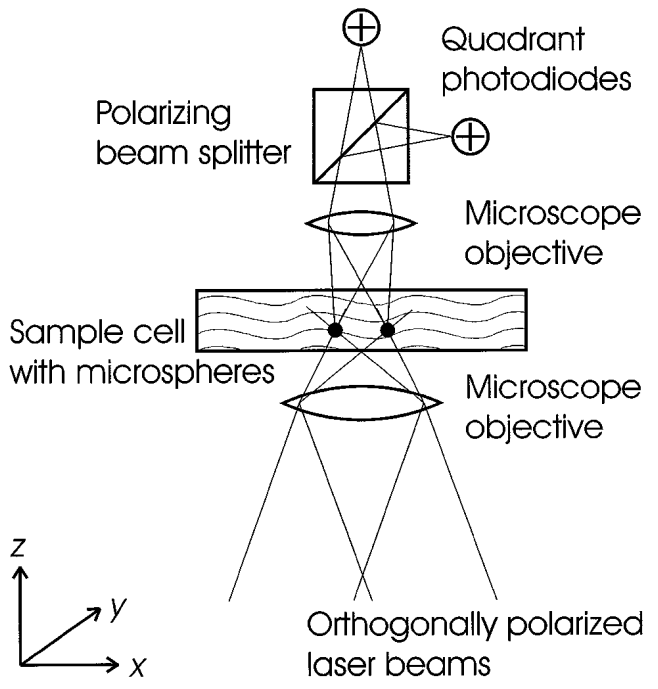

FIG. 1. Schematic diagram of the optical tweezer apparatus. Two orthogonally polarized laser beams are focused into the sample cell, where each of them holds a microsphere. The light scattered from the microspheres is collected with a second microscope objective, separated by a polarizing beam splitter, and focused onto a position sensitive quadrant photodiode. Data points are acquired with $20 \mu \mathrm{sec}$ time resolution and an ultimate position resolution of $\sim 1 \mathrm{~nm}$. The force sensitivity is $\sim 200 \mathrm{f} \mathrm{N} / \sqrt{\mathrm{Hz}}$.

tally obtained autocorrelation functions we also see a second exponential with a different time constant, both with and without a second bead present. This second time constant is typically an order of magnitude longer than $\tau_{x}$, and the corresponding amplitude is about $20 \%$ of the principal exponential. We attribute this second time constant to the motion of the bead along the weaker $z$ axis of the trap, which couples to a small

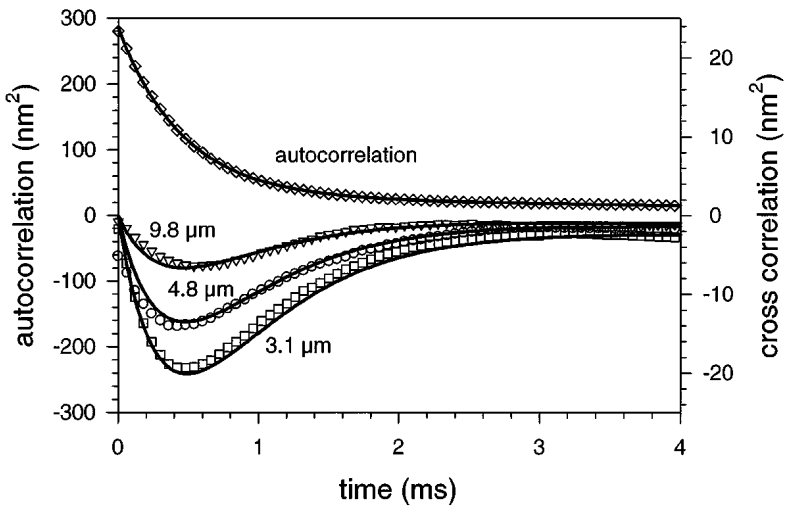

FIG. 2. Longitudinal correlation functions of the position of the two beads. The upper curve shows the autocorrelation function of a single bead in its trap, together with a double exponential fit. The lower curves show the cross-correlation functions of two beads held at separations of 9.8, 4.8, and $3.1 \mu \mathrm{m}$, respectively, together with the theoretically predicted curves, as detailed in the text. Only every third of the experimentally obtained data points is shown. 
degree into the detector signal. This notion was verified by changing the depth of the plane of focus inside the sample cell, thus changing the trap strength in the $z$ direction. The time constant of the second exponential changed accordingly. Since the two time constants differ vastly, the contributions from the motion of the bead along either axis are readily distinguishable. Indeed, a double exponential decay fits the experimentally obtained autocorrelation functions perfectly, yielding the time constants for motion in the $x$ and $z$ axes and a calibration factor for the amplitudes. A similar effect has been observed with localized dynamic light scattering [13].

For a theoretical framework to understand the autoand cross-correlation functions, we utilize the Langevin equation [2] for the stochastic motion of particles in a fluid and external potential. The equations of motion for the particles are

$$
\frac{d \mathbf{R}_{n}}{d t}=\sum_{m=1}^{2} \mathbf{H}_{n m}\left(\mathbf{R}_{n}-\mathbf{R}_{m}\right)\left[-k \mathbf{R}_{m}+\mathbf{f}_{m}(t)\right] .
$$

Brownian forces are represented by randomly fluctuating functions $\mathbf{f}_{m}(t)$ which satisfy the following correlations:

$$
\left\langle\mathbf{f}_{m}(t)\right\rangle=0 ; \quad\left\langle\mathbf{f}_{n}(t) \mathbf{f}_{m}\left(t^{\prime}\right)\right\rangle=2 \mathbf{H}_{n m}^{-1} k_{B} T \delta\left(t-t^{\prime}\right) .
$$

The hydrodynamic interactions of the particles with the surrounding fluid are described by their mobility matrix $\mathbf{H}_{n m}$, which is also known as the Oseen tensor:

$\mathbf{H}_{n n}(\mathbf{R})=\frac{\mathbf{I}}{\zeta} ; \quad \mathbf{H}_{n m}(\mathbf{R})=\frac{1}{8 \pi \eta R}(\mathbf{I}+\hat{\mathbf{R}} \hat{\mathbf{R}})$.

$\zeta=6 \pi \eta a$ is the friction coefficient of a sphere of radius $a$ in a solvent with viscosity $\eta, \mathbf{I}$ denotes the $3 \times 3$ unity matrix, and $\hat{\mathbf{R}}$ is the unity vector parallel to $\mathbf{R}$. Higher-order corrections to the matrix elements in Eq. (3) are small, scaling as $(a / E)^{4}$ for the diagonal elements and $(a / E)^{3}$ for the off-diagonal elements [14]. Under our experimental conditions, these corrections are always smaller than $1 \%$ or $3.5 \%$, respectively. Since the coupling in Eq. (3) is nonlinear, there is no general closed-form solution to Eq. (1). However, since individual beads move only with a rms amplitude of $16 \mathrm{~nm}$ in the trap, $\mathbf{R}_{2}-\mathbf{R}_{1} \approx E \hat{\mathbf{x}}$, and thus to a good approximation $\mathbf{H}_{n m}$ is constant and Eq. (1) is linear.

It is then a straightforward calculation to find the normal coordinates in which Eq. (1) decouples, and then the correlation functions for the vector components $R_{i}$ $(i=x, y, z)$ can be directly calculated:

$$
\begin{aligned}
\left\langle R_{1, i}(t) R_{1, j}(0)\right\rangle & =\left\langle R_{2, i}(t) R_{2, j}(0)\right\rangle \\
& =\delta_{i j} \frac{k_{B} T}{2 k_{i}}\left(e^{-t\left(1+\varepsilon_{i}\right) / \tau_{i}}+e^{-t\left(1-\varepsilon_{i}\right) / \tau_{i}}\right),
\end{aligned}
$$

$$
\begin{aligned}
\left\langle R_{1, i}(t) R_{2, j}(0)\right\rangle & =\left\langle R_{2, i}(t) R_{1, j}(0)\right\rangle \\
& =\delta_{i j} \frac{k_{B} T}{2 k_{i}}\left(e^{-t\left(1+\varepsilon_{i}\right) / \tau_{i}}-e^{-t\left(1-\varepsilon_{i}\right) / \tau_{i}}\right),
\end{aligned}
$$

where the fundamental relaxation time $\tau_{i}=\zeta / k_{i}$ is determined by the trap strength $k_{i}$ and the friction of the bead $\zeta$. The dimensionless parameter $\varepsilon_{i}$ describes the ratio between the mobility of the beads and the strength of the hydrodynamic coupling between them, which amounts to $\varepsilon_{x}=3 a / 2 E$ for motion in the longitudinal axis of the beads and $\varepsilon_{y}=\varepsilon_{z}=3 a / 4 E$ for the transverse axis. In our experiment, a typical value of $\tau_{x}$ was $0.45 \mathrm{~ms}$, which corresponds to a trap stiffness of $18.5 \mathrm{pN} / \mu \mathrm{m}$.

Armed with the analytical expressions for the correlations between the spheres we can now interpret the experimental results. First, we note that the autocorrelation functions in Eq. (4) consist of two exponentials with equal amplitude and time constants that are very close to the fundamental relaxation time of the traps $\tau_{x}$, compared to a single exponential decay with twice the amplitude and a relaxation time $\tau_{x}$ for a single trapped bead in absence of any hydrodynamic interactions. In fact, the change in the autocorrelation functions due to the presence of the second bead turns out to be so small that it is not noticeable in the experimentally obtained autocorrelation functions. However, the split in the time constants dominates the cross-correlation function [Eq. (5)]. Physically, it reflects the asymmetry of the hydrodynamic interaction: Since one sphere tends to drag the other in its wake, correlated fluctuations relax faster than anticorrelated fluctuations, in which the fluid between the spheres must be displaced.

Since $\tau_{x}$ is known from the trap calibration, the crosscorrelation function [Eq. (5)] can be predicted exactly with no free parameters. A small correction accounting for the coupling in the $z$ direction can also be computed. This correction is calculated with Eq. (5) using the time constant $\tau_{z}$ and the amplitude of the secondary exponential from the autocorrelation functions. It mostly affects the shape of the tails of the curves at longer times, while it remains below $3 \%$ near the minimum. The result of this procedure is shown for three representative curves together with the actual experimental data in Fig. 2. In the transverse direction (data not shown) the cross-correlation functions are in quantitative agreement with Eq. (5), verifying the directional dependence of $\varepsilon$.

The cross-correlation curves exhibit a time-delayed anticorrelation with a pronounced minimum at $t_{\min }=$ $\left(\tau_{i} / 2 \varepsilon_{i}\right) \ln \left[\left(1+\varepsilon_{i}\right) /\left(1-\varepsilon_{i}\right)\right] \approx \tau_{i}$. The depth of the minimum

$$
\left\langle R_{1, i}\left(\tau_{i}\right) R_{2, i}(0)\right\rangle \approx-\frac{1}{e} \frac{k_{B} T}{k_{i}} \sinh \left(\varepsilon_{i}\right) \approx-\frac{1}{e} \frac{k_{B} T}{k_{i}} \varepsilon_{i}
$$




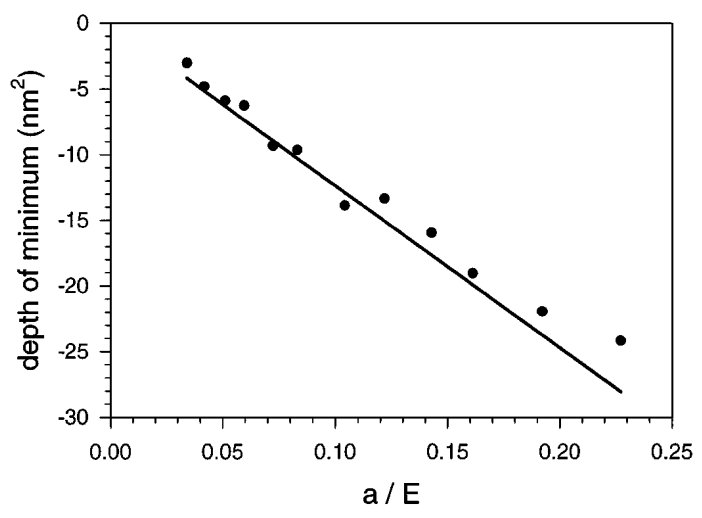

FIG. 3. Depth of the minimum in the longitudinal crosscorrelation functions as a function of the ratio between the radius of the spheres $a$ and their average separation $E$. The prediction from the linear approximation in Eq. (6) is shown as the straight line for comparison. No corrections for motion along the $z$ axis have been included.

is in good approximation proportional to the strength of the hydrodynamic coupling $\varepsilon$, which in turn is inversely proportional to the separation between the beads. The shapes of the cross-correlation curves are almost selfsimilar; if we normalize them by the depth of their minimum, they are nearly indistinguishable. The error stemming from the linearizations in Eq. (6) is smaller than $2 \%$ for all data points.

Measuring directly the depth of the minimum in the longitudinal cross correlation as a function of $a / E$ for a large number of different separations between the beads confirms the expected linear relationship from Eq. (6), as depicted in Fig. 3. Since the correction for motion along the $z$ axis is negligible on such a short time scale, no such corrections have been included here. Corrections due to the slight mismatch of spring constants are of second order and are neglected. The last data point in Fig. 3 is in a statistically significant disagreement with the theoretical prediction, which corrections to the various linearizations in our model cannot account for. However, for that point the gap between the spheres is as small as the wavelength of the light used for the traps. Thus the deviation is likely a systematic error due to double scattering from the spheres or optical near-field effects.

In conclusion, we have directly measured the effects of hydrodynamic coupling on the dynamics of two particles held in potential wells and shown that the observed timedelayed anticorrelation between the particles can be understood in the framework of Langevin dynamics. The hydrodynamically coupled spheres also serve as a general model system and might help in understanding microscopic biological dynamics. It is conceivable that proteins, organelles, or even cells use hydrodynamic correlations to synchronize signaling or other collective behavior.

We thank John Doyle, John Brady, Noel Corngold, and Michael Brenner for helpful discussions. This work was supported by NSF CAREER Grant No. PHY-9722417.

[1] T. G. M. van de Ven, Colloidal Hydrodynamics (Academic, London, 1989).

[2] M. Doi and S.F. Edwards, The Theory of Polymer Dynamics (Oxford Press, Oxford, 1994).

[3] J. G. de la Torre and V. A. Bloomfield, Q. Rev. Biophys. 14, 81 (1981).

[4] S. Hayward and N. Go, Annu. Rev. Phys. Chem. 46, 223 (1995).

[5] T. Ichiye and M. Karplus, Proteins Struct. Funct. Genet. 11, 205 (1991).

[6] A. Kitao, F. Hirata, and N. Go, J. Chem. Phys. 158, 447 (1991).

[7] D. Brune and S. Kim, Proc. Natl. Acad. Sci. U.S.A. 91, 2930 (1994).

[8] J. C. Crocker and D. G. Grier, Phys. Rev. Lett. 73, 352 (1994).

[9] T. Sugimoto, T. Takahashi, H. Itoh, S. Sato, and A. Muramatsu, Langmuir 13, 5528 (1997).

[10] J. C. Crocker, J. Chem. Phys. 106, 2837 (1997).

[11] E. M. Purcell, Am. J. Phys. 45, 3 (1977).

[12] B.C. Kuo, Automatic Control Systems (Prentice-Hall, Englewood Cliffs, NJ, 1987).

[13] R. Bar-Ziv, A. Meller, T. Tlusty, E. Moses, J. Stavans, and S. A. Safran, Phys. Rev. Lett. 78, 154 (1997).

[14] G. K. Batchelor, J. Fluid Mech. 74, 1 (1976). 